\title{
Latest Results from the AMS Experiment on the International Space Station
}

\author{
Zhili Weng ${ }^{\dagger, *}$ \\ Massachusetts Institute of Technology, Cambridge, USA \\ E-mail: zhili.weng@cern.ch
}

The Alpha Magnetic Spectrometer is a general-purpose particle physics detector operating on the International Space Station. Precision measurements by AMS of the cosmic-ray elementary particle fluxes and nuclei fluxes reveal new unexpected phenomena. The positron flux exhibits a significant excess starting from $25.2 \pm 1.8 \mathrm{GeV}$ followed by a sharp drop-off above $284_{-64}^{+91} \mathrm{GeV}$, consistent with a primary source of cosmic-ray positrons from either dark matter collisions or new astrophysical sources. The different behavior of the cosmic-ray electron flux and positron flux shows that most high energy electrons originate from different sources than high energy positrons. Intriguingly, the positron flux and the antiproton flux have strikingly similar behavior at high energies. New observations from AMS on cosmic nuclei show that primary cosmic-ray $\mathrm{He}, \mathrm{C}$, and $\mathrm{O}$ have an identical rigidity dependence above $60 \mathrm{GV}$ and deviate from a single powerlaw above $200 \mathrm{GV}$. Unexpectedly, the primary $\mathrm{Ne}, \mathrm{Mg}$, and $\mathrm{Si}$ also have an identical rigidity dependence above $86.5 \mathrm{GV}$, but they are different from that of $\mathrm{He}, \mathrm{C}$, and $\mathrm{O}$. This shows that primary cosmic rays have at least two distinct classes of rigidity dependence. Above $30 \mathrm{GV}$, secondary cosmic nuclei $\mathrm{Li}, \mathrm{Be}$, and $\mathrm{B}$ have identical rigidity dependence which is distinctly different from those of primary cosmic rays. The results from AMS on many different types of cosmic rays are not explained by the current theoretical models and provide unique input to the understanding of the origins and evolution of cosmic rays in the galaxy.

40th International Conference on High Energy physics - ICHEP2020

July 28 - August 6, 2020

Prague, Czech Republic (virtual meeting)

\footnotetext{
$\dagger$ on behalf of the AMS Collaboration

*Speaker
} 


\section{The Alpha Magnetic Spectrometer}

The Alpha Magnetic Spectrometer (AMS) [1] is a precision general-purpose particle physics detector on the International Space Station (ISS) for the studies of the origin of dark matter, antimatter, and cosmic rays as well as to explore new physics phenomena. The AMS detector consists of a permanent magnet and an array of particle detectors to measure the velocity $\beta=v / c$, energy $E$, momentum $P$, charge $Z$, and rigidity $R=P / Z$ of traversing particles and nuclei. The layout of the AMS detector is illustrated in Figure 1. The Transition Radiation Detector (TRD) is located at the top of the AMS. Two orthogonal planes of Time of Flight counters above the magnet bore (Upper TOF) and another two orthogonal planes below the magnet bore (Lower TOF). There are a total of 9 precision silicon tracker layers located within the magnet bore and above and below the magnet. The Anti-Coincidence Counters (ACC or Veto) surround the tracker within the magnet bore. The Ring Imaging Cherenkov counter (RICH) is below the Lower TOF, and below that is the Electromagnetic Calorimeter (ECAL). AMS was installed on the ISS on 19 May 2011, since then, AMS has functioned reliably and the properties of the detector are continuously monitored. The detailed description of the AMS detector and its operations are summarized in Ref.[1].

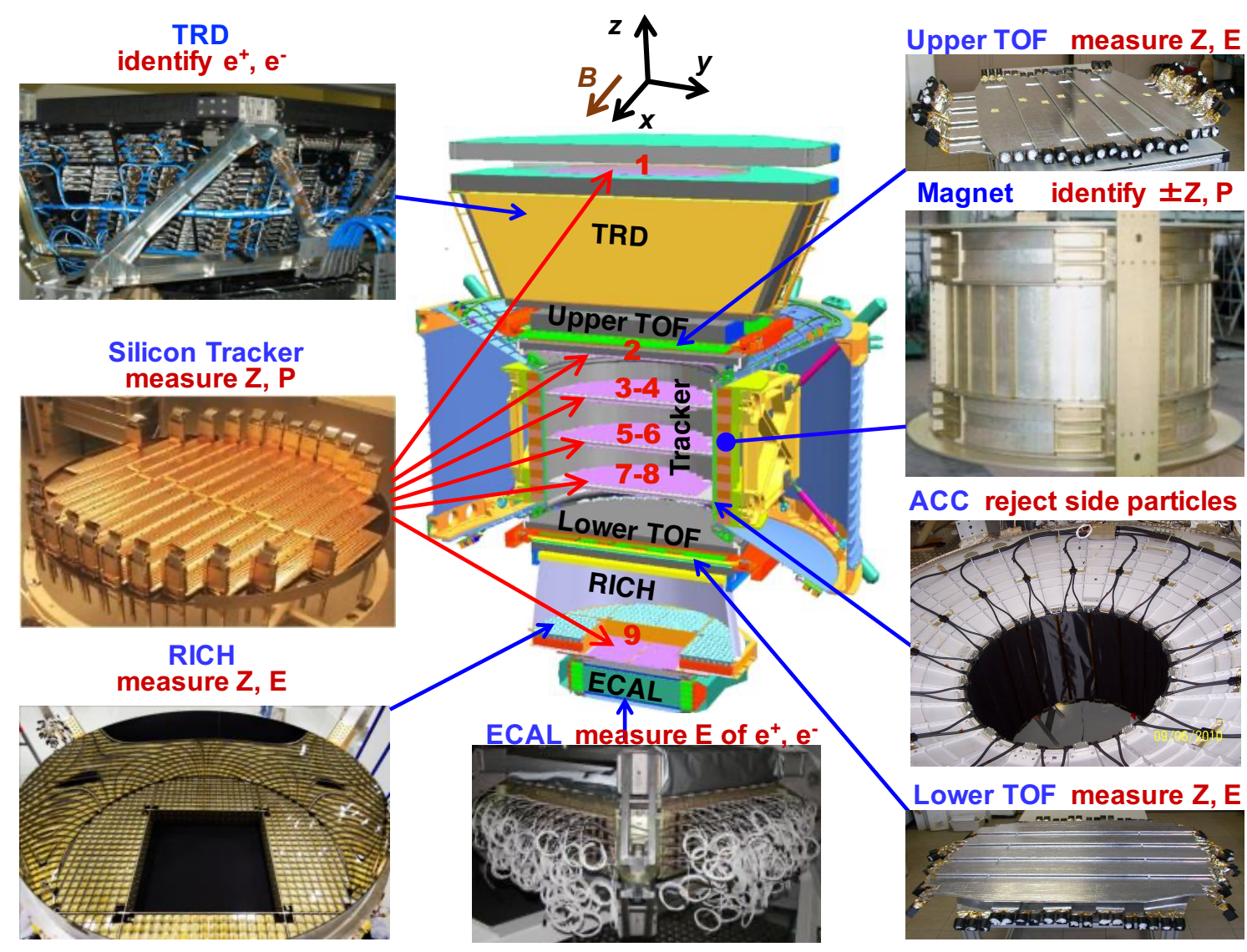

Figure 1: The AMS detector showing the main elements and their functions. 


\section{Precision Measurements of Cosmic Elementary Particles}

Precision measurements by AMS on the fluxes of protons, electrons, positrons, and antiprotons over an extended energy range [1] provide crucial information in the study of cosmic-ray elementary particles and the search for new physics phenomena in the cosmos.

\subsection{Origins of Cosmic Positrons and Electrons}

There have been widespread interests and explanations on the origin of cosmic-ray positrons and electrons, with different predictions for the behavior of their fluxes towards high energy [2]. The latest AMS results on the precision measurements of positron spectrum (i.e., the flux scaled by $\tilde{E}^{3}, \tilde{E}^{3} \Phi_{e^{+}}$, where $\tilde{E}$ is the spectrally weighted mean energy in each bin calculated for a flux $\propto E^{-3}$ ) up to $1 \mathrm{TeV}$ and the electron spectrum $\left(\tilde{E}^{3} \Phi_{e^{-}}\right)$up to $1.4 \mathrm{TeV}$ reveal complex behavior of the two spectra [3]. As presented in Figure 2, in the entire energy range, the electron spectrum and the positron spectrum have distinctly different magnitudes and energy dependences.

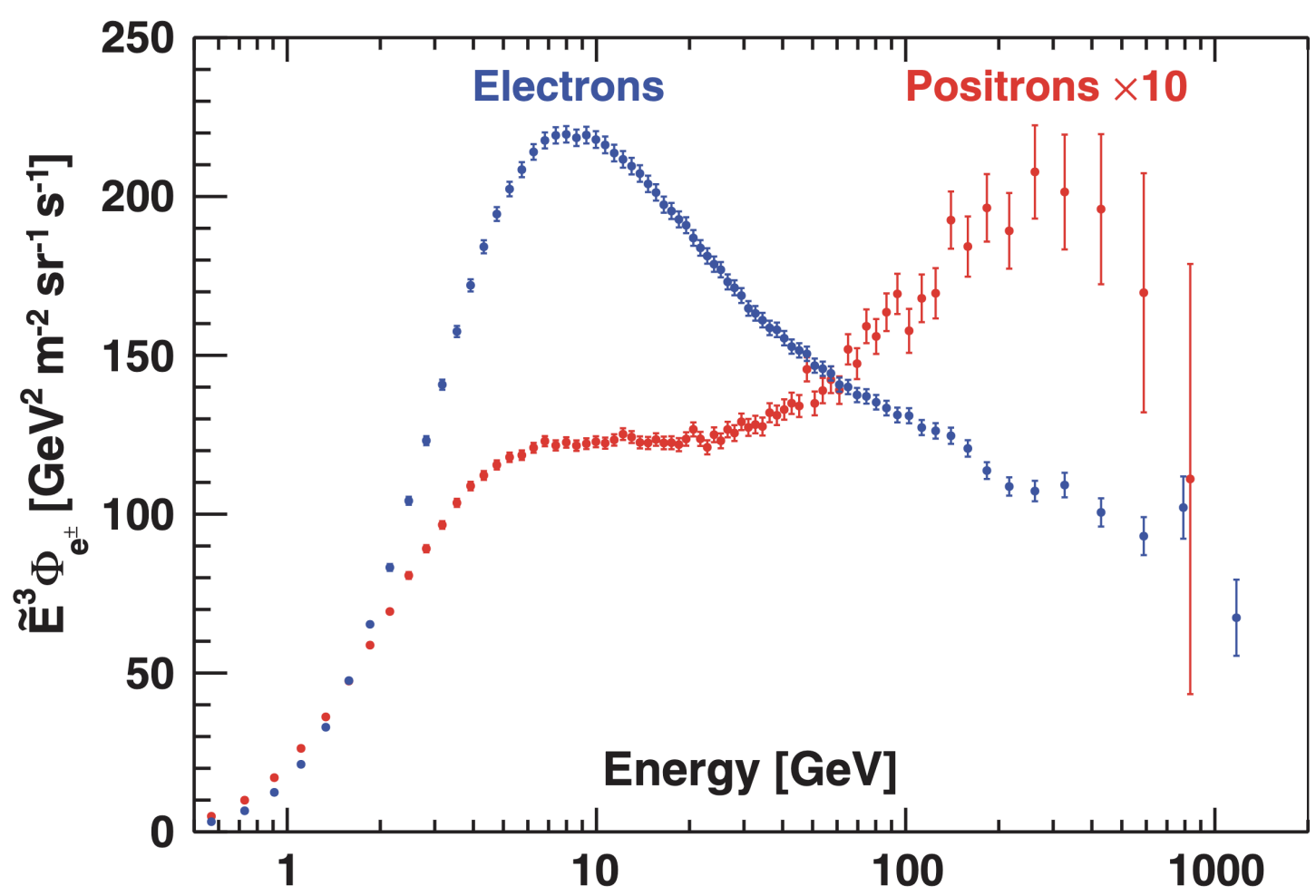

Figure 2: The AMS positron spectrum (red data points) and electron spectrum (blue data points). The electron spectrum and the positron spectrum have distinctly different magnitudes and energy dependences

The positron spectrum shows a significant excess starting from $25.2 \pm 1.8 \mathrm{GeV}$, it then reaches a maximum at $284_{-64}^{+91} \mathrm{GeV}$, followed by a sharp drop-off [3]. At energy starting from $\sim 10 \mathrm{GeV}$, the positron flux by far exceeds the contribution from secondary positrons [4]. The complex behavior of the positron flux is consistent with the existence of a new source of high energy positrons. The positron flux can be parametrized as the sum of a diffuse term and a source term: $\Phi_{e^{+}}(E)=\frac{E^{2}}{\hat{E}^{2}}\left[C_{d}\left(\hat{E} / E_{1}\right)^{\gamma_{d}}+C_{s}\left(\hat{E} / E_{2}\right)^{\gamma_{s}} \exp \left(-\hat{E} / E_{s}\right)\right]$. The diffuse term is characterized by a 
normalization factor $C_{d}$ and a spectral index $\gamma_{d}$. The source term is described by a normalization factor $C_{s}$, a spectral index $\gamma_{s}$, with an exponential cutoff energy $E_{s} . \hat{E}$ denotes the energy of particles in the interstellar space [3]. The result of the fit is presented in Figure 3 (a). As seen, the diffuse term dominates at low energies and then gradually vanishes with increasing energy. The source term, with the exponential cutoff energy $E_{S}=810_{-180}^{+310} \mathrm{GeV}$, describes the behavior of the positron spectrum at high energies.
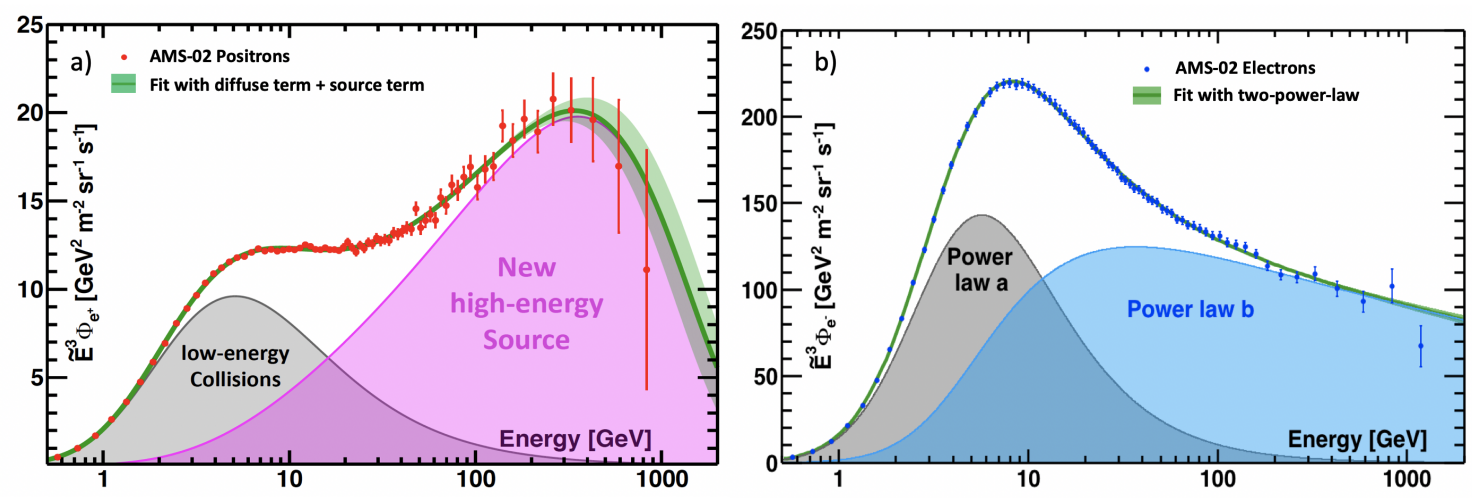

Figure 3: (a) The fit of two components (green line) to the positron flux in the energy range [0.5 - 1000] GeV together with the 68\% C.L. interval (green band). The source term contribution is represented by the magenta area, and the diffuse term contribution by the grey area. (b) The two-power-law fit to the electron flux data in the energy range [0.5 - 1400] GeV with the 68\% C.L. (green band). The two power-law components $a$ and $b$ are represented by the gray and blue areas, respectively.

The electron spectrum is distinctly different from the positron spectrum. Detailed analyses[3] show that the electron flux exhibits a significant excess starting from $42.1_{-5.2}^{+5.4} \mathrm{GeV}$, but the nature of this excess is different from the positron flux excess. Contrary to the positron flux, at over $5 \sigma$ level, the electron flux does not show an exponential energy cutoff below $1.9 \mathrm{TeV}$. The AMS electron flux is well described by the sum of two power-law components: $\Phi_{e^{-}}(E)=\frac{E^{2}}{\hat{E}^{2}}[1+$ $\left.\left(\hat{E} / E_{t}\right)^{\Delta \gamma_{t}}\right]^{-1}\left[C_{a}\left(\hat{E} / E_{a}\right)^{\gamma_{a}}+C_{b}\left(\hat{E} / E_{b}\right)^{\gamma_{b}}\right]$, as presented in Figure $3(\mathrm{~b})$. These functions are very different in shape and magnitude from those describing the positron flux. The different behavior of the cosmic-ray electrons and positrons measured by AMS is clear evidence that most high energy electrons originate from different sources than high energy positrons.

The data from AMS show that, at high energies, positrons predominantly originate either from dark matter annihilation or from other astrophysical sources. As an example, Figure 4 shows the comparison of the AMS data with a dark matter model based on Ref. [4, 5] with a dark matter particle mass of $1.2 \mathrm{TeV}$. More statistics at high energies are required to verify the agreement and to understand the behavior of the positron spectrum beyond the cutoff energy. Positrons and electrons may also be produced and accelerated from astrophysical sources like pulsars [2]. Point sources like pulsars will imprint a higher degree of anisotropy on the arrival directions of high energy positrons and electrons compared to a smooth dark matter halo. Current AMS measurements [6] show that the incoming directions of positrons and electrons are consistent with isotropic. With more data, AMS will continue to improve the accuracy and the energy reach of the measurements on positrons and electrons so as to determine their origin in the cosmos. 


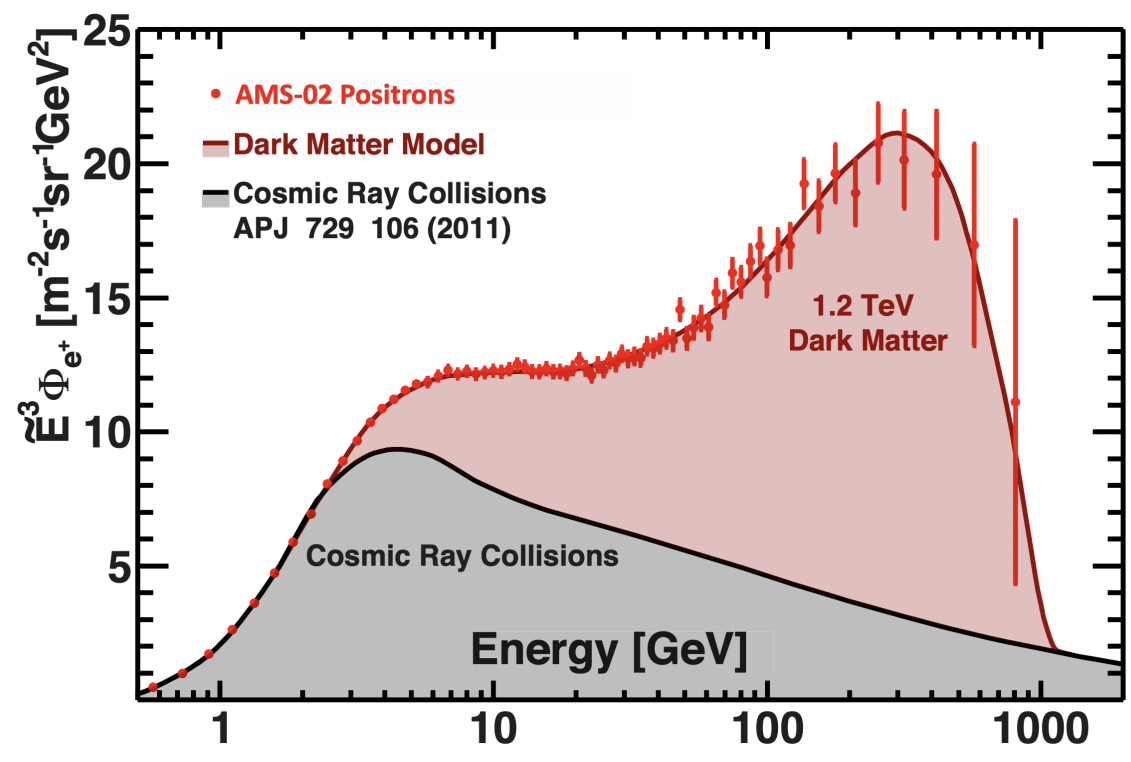

Figure 4: Comparison of the AMS data with predictions of a dark matter model based on Ref. [4, 5]. More statistics at high energies are required to verify the agreement and to understand the behavior of the positron spectrum beyond the cutoff energy.

\subsection{Properties of Protons and Antiprotons}

Protons are the most abundant charged cosmic rays. Knowledge of the rigidity dependence of the proton flux is crucial in understanding the origin, acceleration, and propagation of cosmic rays [7]. Different variations of the proton flux have been reported by recent measurements [8]. Figure 5 (a) shows the latest AMS measurements [1,9] of the proton flux compared with recent measurements. As seen, the AMS results provide accurate information on the proton flux in the energy range from $1 \mathrm{GV}$ to $1.8 \mathrm{TV}$. Figure 5 (b) shows the AMS proton flux multiplied by $\tilde{R}^{2.7}$ as a function of rigidity, where $\tilde{R}$ is the spectrally weighted mean rigidity in each bin calculated for a flux $\propto R^{-2.7}$. To describe the changing behavior of the proton flux at high rigidity, we use the function $\Phi=C\left(\frac{R}{45 \mathrm{GV}}\right)^{\gamma}\left[1+\left(\frac{R}{R_{0}}\right)^{\Delta \gamma / s}\right]^{s}$. As shown in Figure $5(\mathrm{~b})$, The proton flux deviates from a single power law and harden $(\Delta \gamma>0)$ progressively above $200 \mathrm{GV}$.

Measurements of the cosmic ray antiproton flux are important as the sensitivity of antiprotons to new physics phenomena in the cosmos is complementary to the sensitivity of the measurements of cosmic-ray positrons. The latest AMS measurements of the antiproton flux from 1 to $525 \mathrm{GV}$ reveal new properties of cosmic-ray antiprotons $[1,10]$. Most surprisingly, the AMS measurements show that the positron spectrum and the antiproton spectrum have strikingly similar behavior at high energies, as presented in Figure 6 (a). In the range $[60-525] \mathrm{GeV}$, the positron-to-antiproton flux ratio is consistent with a constant, as presented in Figure 6 (b). The identical behavior of positron flux and antiproton flux at this energy range suggests a possible common source of high energy positrons and antiprotons. While antiprotons are not produced by pulsars, dark matter annihilation may produce antiprotons with an energy cutoff in the spectrum at high rigidity. The continuation of AMS data taking through the life of the Space Station will provide an important confirmation of the origin of high energy positrons and antiprotons. 

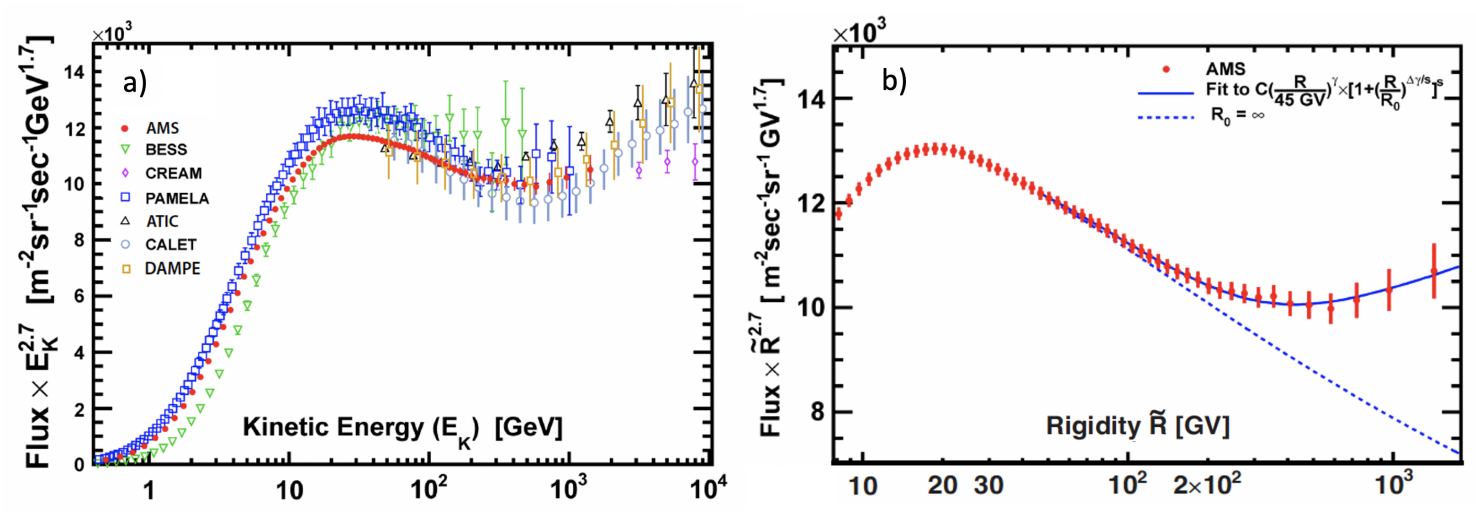

Figure 5: (a) The AMS proton flux multiplied by $E_{K}^{2.7}$ together with other recent measurements. (b) The AMS proton flux multiplied by $\tilde{R}^{2.7}$ together with the fit to the data. The proton flux deviates from a single power law and harden progressively above $200 \mathrm{GV}$.
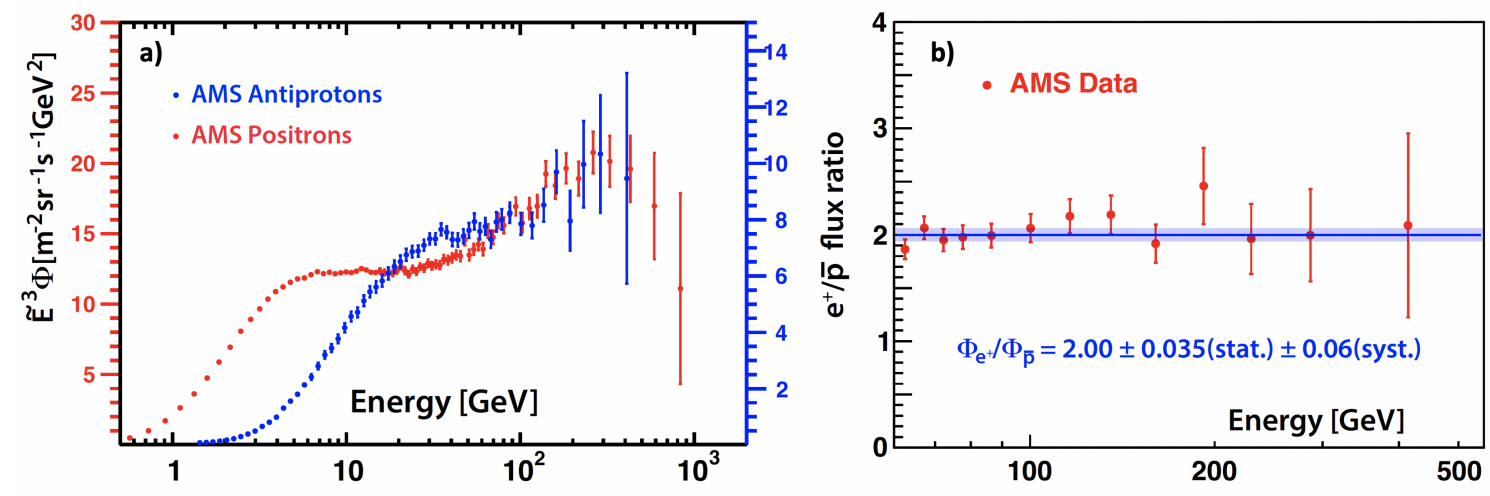

Figure 6: (a) Comparison of the AMS positron spectrum $\left(\tilde{E}^{3} \Phi_{e^{+}}\right.$, red data points, left axis) and antiproton spectrum $\left(\tilde{E}^{3} \Phi_{\bar{p}}\right.$, blue data points, right axis). They exhibit striking similarity at high energy. (b) The positron-to-antiproton flux ratio. In the range $[60-525] \mathrm{GeV}$ with the result of the fit with a constant value.

\section{Precision Measurements of Cosmic Nuclei Fluxes}

There are several instruments in the AMS detector which independently measure the charge of cosmic rays. Figure 7 shows all the charged cosmic nuclei measured by the TOF and the tracker. Cosmic nuclei up to iron and beyond are clearly identified and are studied in detail by AMS.

\subsection{Properties of Primary Cosmic-Ray Nuclei}

Primary cosmic-ray nuclei are believed to be produced and accelerated by astrophysical sources like supernova remnants. Precision measurements of their spectra provide important information on the origin, acceleration, and propagation of galactic cosmic rays.

Helium, carbon, and oxygen are the most abundant primary cosmic-ray nuclei. The AMS results on the $\mathrm{He}, \mathrm{C}$, and $\mathrm{O}$ spectra [1,9] are presented in Figure 8 (a). These results reveal an identical rigidity dependence of these three fluxes above $60 \mathrm{GV}$. In particular, they all deviate from a single power-law and harden progressively from $\sim 200 \mathrm{GV}$. These new observations have generated new developments in cosmic-ray models $[11,12]$. 


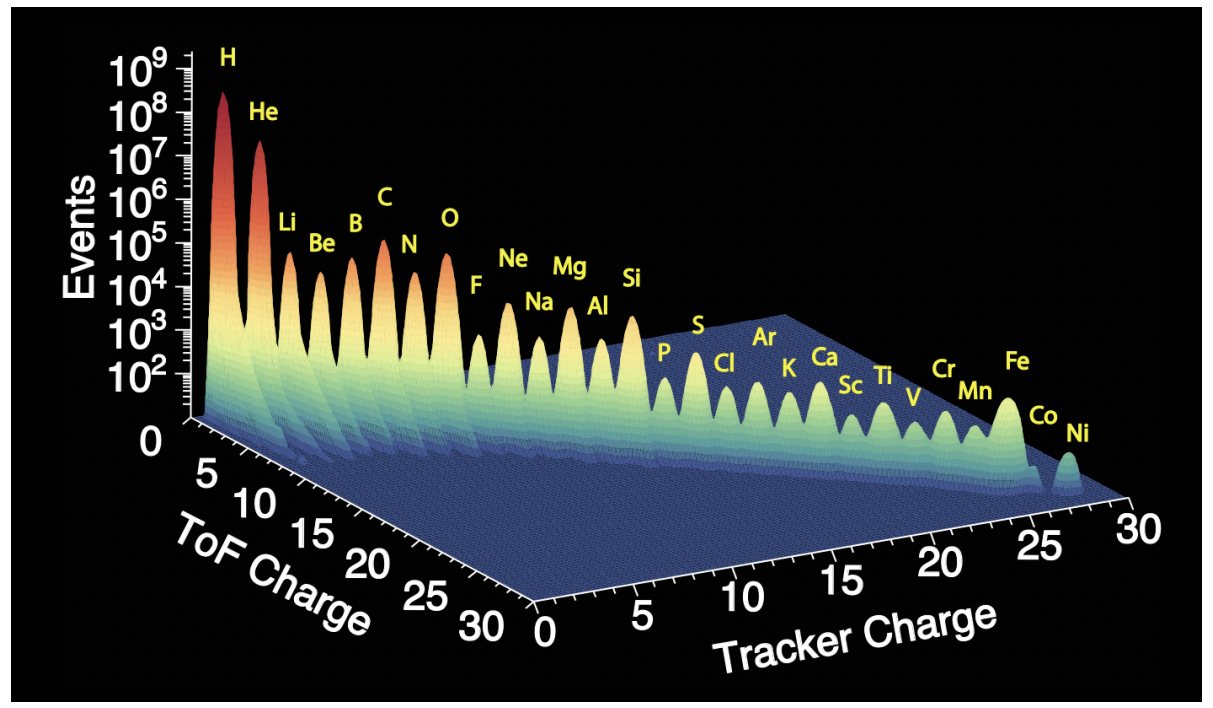

Figure 7: The charge measurement by AMS using the TOF and the tracker. Cosmic nuclei up to iron and beyond are clearly identified and are studied in detail by AMS.
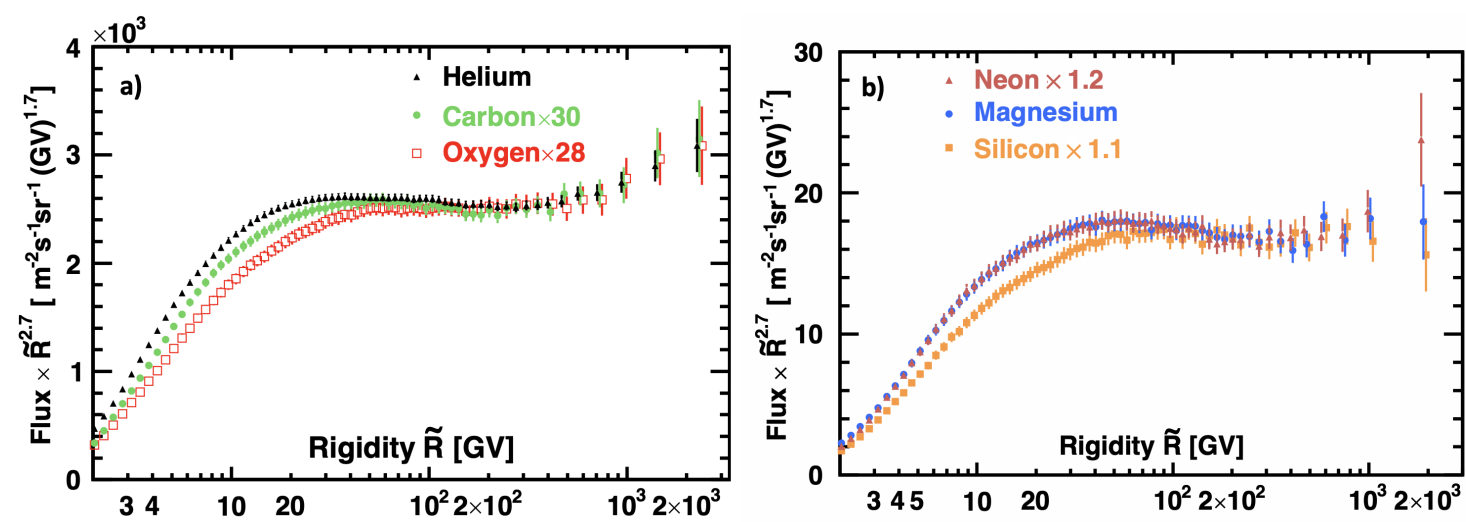

Figure 8: The AMS measurements of primary cosmic nuclei fluxes multiplied by $\tilde{R}^{2.7}$ for: (a) He, C, and O nuclei, and (b) $\mathrm{Ne}, \mathrm{Mg}$, and Si nuclei.

Neon, magnesium, and silicon are assumed to be primary nuclei. Detailed comparison of the differences in the rigidity dependences of $\mathrm{Ne}, \mathrm{Mg}$, and $\mathrm{Si}$ with those of $\mathrm{He}, \mathrm{C}$, and $\mathrm{O}$ provide new insights into the origin and propagation of cosmic rays [11,13]. The latest AMS measurements of the $\mathrm{Ne}, \mathrm{Mg}$, and Si fluxes in the rigidity range from $2.15 \mathrm{GV}$ to $3.0 \mathrm{TV}[1,14]$ are presented in Figure 8 (b). These three fluxes have an identical rigidity dependence above $86.5 \mathrm{GV}$, and all three fluxes deviate from a single power-law with rigidity above $\sim 200 \mathrm{GV}$.

Unexpectedly, the new observations from AMS show that the rigidity dependence of $\mathrm{Ne}, \mathrm{Mg}$, and $\mathrm{Si}$ is different from that of $\mathrm{He}, \mathrm{C}$, and $\mathrm{O}$. Above $86.5 \mathrm{GV}$, the ratios of the $\mathrm{Ne}, \mathrm{Mg}$, and $\mathrm{Si}$ fluxes to the $\mathrm{O}$ flux are all consistent with a power-law function $\frac{\Phi_{\mathrm{Ne}, \mathrm{Mg}, \mathrm{Si}}}{\Phi_{\mathrm{O}}}=(R / 86.5 \mathrm{GV})^{\delta}$ with the average value of the spectral indices $\langle\delta\rangle=-0.045 \pm 0.008$. The difference of $\langle\delta\rangle$ from zero by more than $5 \sigma$ shows that the $\mathrm{Ne}, \mathrm{Mg}$, and $\mathrm{Si}$ belong to a different class of primary cosmic rays than $\mathrm{He}, \mathrm{C}$, and $\mathrm{O}$. We use the function $\Phi=C\left(\frac{R}{45 \mathrm{GV}}\right)^{\gamma}\left[1+\left(\frac{R}{R_{0}}\right)^{\Delta y / s}\right]^{S}$ to compare the rigidity dependence of 


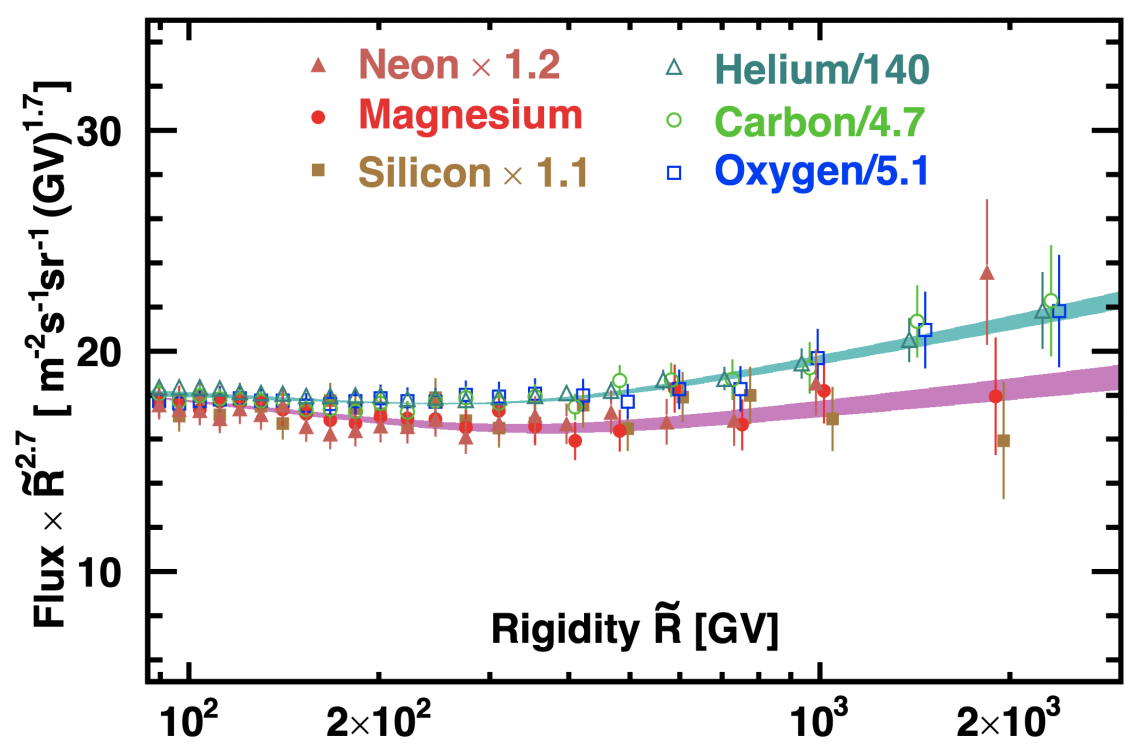

Figure 9: Above $86.5 \mathrm{GV}$, the rigidity dependence of the $\mathrm{Ne}, \mathrm{Mg}$, and $\mathrm{Si}$ fluxes is different from the rigidity dependence of the $\mathrm{He}, \mathrm{C}$, and $\mathrm{O}$ fluxes, revealing that primary cosmic rays have at least two distinct classes of rigidity dependence.

$\mathrm{He}, \mathrm{C}$ and $\mathrm{O}$, to that of $\mathrm{Ne}, \mathrm{Mg}$ and $\mathrm{Si}$, as illustrated in Figure 9. These results show that primary cosmic rays have at least two distinct classes of rigidity dependence.

\subsection{Properties of Secondary Cosmic-Ray Nuclei}

Lithium, beryllium, and boron nuclei are secondary cosmic rays produced by the collisions of the primary nuclei with the interstellar media. Knowledge of their spectra provides crucial information on the propagation of cosmic rays as well as the properties of the interstellar media.

Precision measurements from AMS on $\mathrm{Li}, \mathrm{Be}$, and B [1,9] reveal new and unexpected observations. As shown in Figure 10, the three secondary fluxes have an identical rigidity dependence above $30 \mathrm{GV}$ and deviate from a single power-law above $200 \mathrm{GV}$ in an identical way. The rigidity dependence of secondary cosmic ray fluxes is distinctly different from those of primary cosmic ray fluxes. To examine the difference between the rigidity dependence of primary and secondary cosmic rays, the ratios of the $\mathrm{Li}, \mathrm{Be}$, and $\mathrm{B}$ fluxes to the $\mathrm{C}$ and $\mathrm{O}$ fluxes were computed [9]. These secondary to primary flux ratios were then fitted using a single power-law function, $\Phi_{S} / \Phi_{P} \propto R^{\Delta}$, in two non-overlapping rigidity intervals to obtain the detailed variations of the spectral indices $\Delta$. As shown in Figure 11, at high rigidities the secondary cosmic rays harden more than the primary cosmic rays with over $5 \sigma$ significance. These new observations reveal new properties of cosmic-ray propagation in the Galaxy [15].

These unexpected results together with ongoing AMS measurements of cosmic-ray isotopic composition $[1,16]$ and heavier elements in cosmic rays will enable us to determine how many classes of rigidity dependence exist in both primary and secondary cosmic rays and provide important information for the development of theoretical models. 


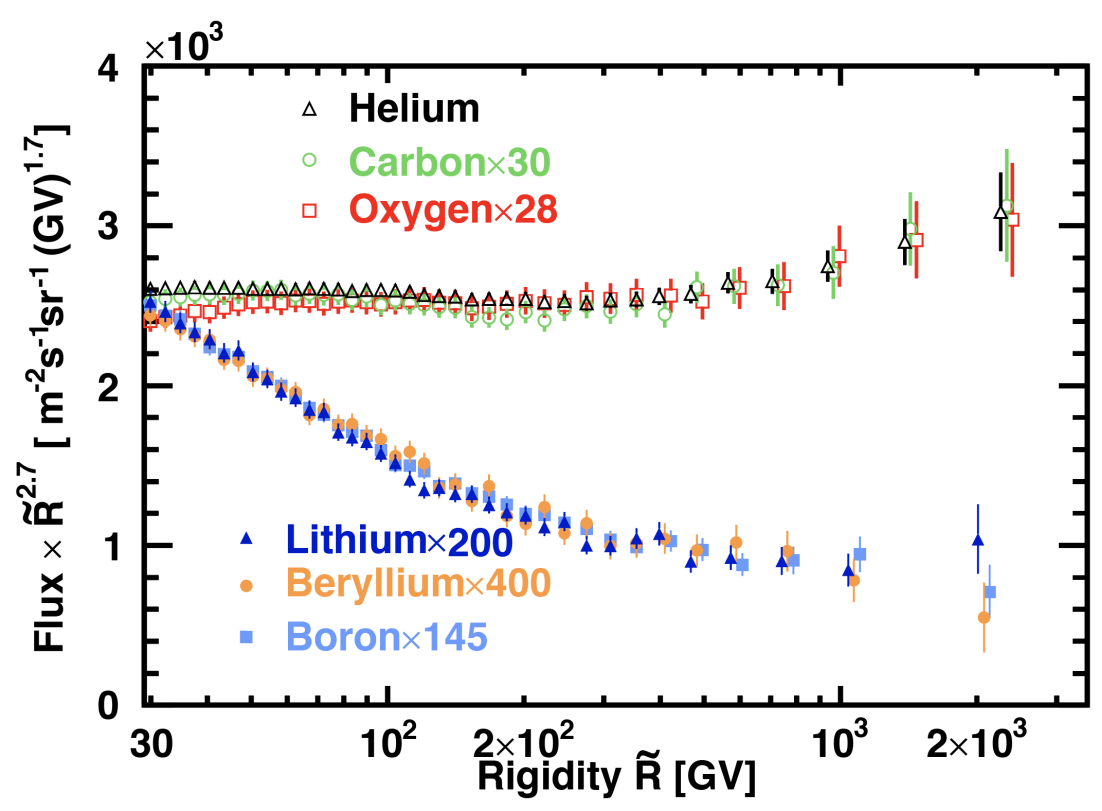

Figure 10: Comparison of the secondary cosmic-ray Li, Be, and B fluxes with the AMS primary cosmic-ray $\mathrm{He}, \mathrm{C}$, and O fluxes multiplied by $\tilde{R}^{2.7}$ above $30 \mathrm{GV}$. The three secondary fluxes have an identical rigidity dependence above $30 \mathrm{GV}$ and deviate from a single power-law above $200 \mathrm{GV}$ in an identical way.
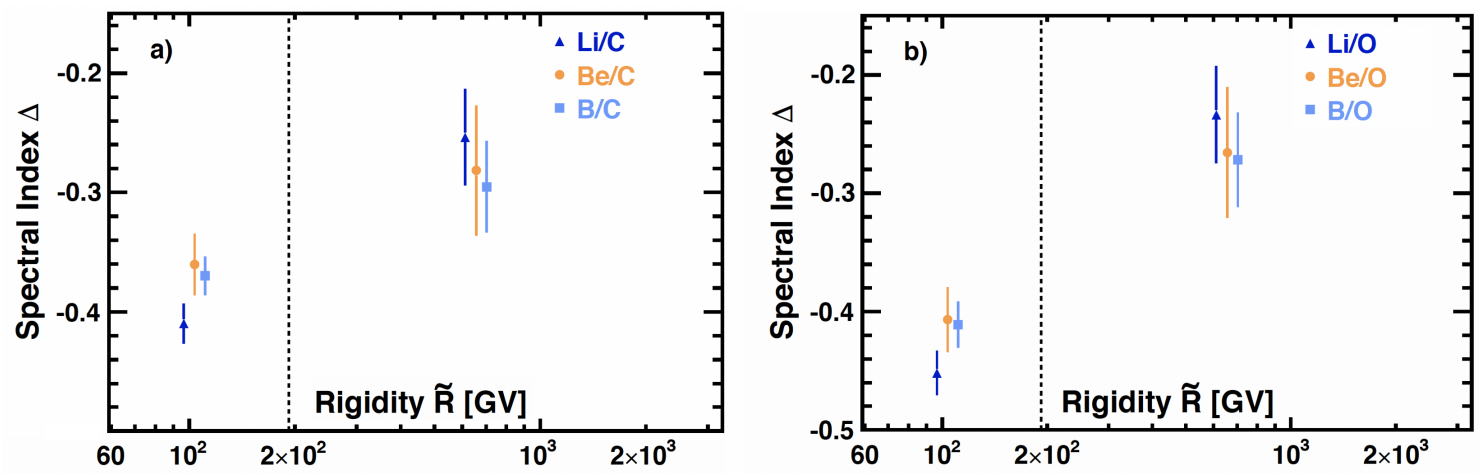

Figure 11: The AMS secondary to primary flux ratio spectral indices $\Delta$ for (a) $\mathrm{Li} / \mathrm{C}, \mathrm{Be} / \mathrm{C}$, and $\mathrm{B} / \mathrm{C}$; (b) $\mathrm{Li} / \mathrm{O}, \mathrm{Be} / \mathrm{O}$, and $\mathrm{B} / \mathrm{O}$. At high rigidities the secondary cosmic rays harden more than the primary cosmic rays with over $5 \sigma$ significance.

\section{Summary}

The latest AMS results on the fluxes of elementary particles and primary and secondary nuclei provide precise and unexpected information. They cannot be explained by the current understanding and call for new comprehensive models of cosmic rays. The accuracy and characteristics of the data, simultaneously from many different types of cosmic rays, provide unique input to the understanding of the origin and evolution of cosmic rays in the galaxy. AMS will continue collecting data with unique precision and energy reach through the life of the Space Station, to explore the physics of complex anti-matter, the physics of dark matter, and the physics of cosmic-ray nuclei across the periodic table. 


\section{References}

[1] M. Aguilar et al., Phys. Rep. In Press, (2020). DOI: 10.1016/j.physrep.2020.09.003

[2] See, for example, M. S. Turner and F. Wilczek, Phys. Rev. D 421001 (1990); J. Ellis, AIP Conf. Proc. 516, 21 (2000); M. Cirelli, R. Franceschini, and A. Strumia, Nucl. Phys. B 800, 204 (2008); P. Blasi. Phys. Rev. Lett. 103051104 (2009); T. Linden and S. Profumo, Astrophys. J. 772, 18 (2013); P. Mertsch and S. Sarkar, Phys. Rev. D 90, 061301 (2014); R. Cowsik, B. Burch, and T. Madziwa-Nussinov, Astrophys. J. 786, 124 (2014);

[3] M. Aguilar et al., Phys. Rev. Lett. 122, 041102 (2019), M. Aguilar et al., Phys. Rev. Lett. 122, 101101 (2019), Z. Weng on behalf of AMS, Presented at this conference, PoS(ICHEP2020) 615.

[4] I. V. Moskalenko and A. W. Strong, Astrophys. J. 493, 694 (1998); R. Trotta, G. Johannesson, I. Moskalenko, T. Porter, R. Ruiz de Austri, and A. Strong, Astrophys. J. 729, 106 (2011).

[5] J. Kopp, Phys. Rev. D 88, 076013 (2013);

[6] M.A. Velasco on behalf of AMS, Presented at this conference, PoS(ICHEP2020) 613.

[7] See, for example, P. Blasi, Braz. J. Phys. 44, 426 (2014); P. Blasi, Astron. Astrophys. Rev., 21, 70 (2013).

[8] For the ATIC experiment see A. D. Panov et al., Bull. Russian Acad. Sci. 73, 564 (2009); For the BESS experiment see K. Abe et al., Phys. Rev. Lett. 108, 051102 (2012); For the CREAM experiment see Y. S. Yoon et al., Astrophys. J. 728, 122 (2011); For the PAMELA experiment see O. Adriani, et al., Astrophys. J. 765, 91 (2013); For the CALET experiment see O. Adriani, et al., Phys. Rev. Lett. 122, 181102 (2019); For the DAMPE experiment see Q. An, et al., Sc. Adv. 5, no. 9, eaax3793 (2019).

[9] M. Paniccia on behalf of AMS, Presented at this conference, PoS(ICHEP2020) 600.

[10] Z. Tang on behalf of AMS, Presented at this conference, PoS(ICHEP2020) 611.

[11] M.J. Boschini et al., Astrophys. J. 840, 115 (2017); M.J. Boschini et al., Astrophys. J. 858, 61 (2018).

[12] C. Evoli, R. Aloisio, and P. Blasi, Phys. Rev. D 99, 103023 (2019); M.J. Boschini et al., Astrophys. J. 889, 167 (2020); N. Weinrich, Y. Génolini, M. Boudaud, L. Derome, and D. Maurin, submitted to Astron. Astrophys. (2020).

[13] G. Jóhannesson et al., Astrophys. J. 824, 16 (2016).

[14] M. Aguilar et al., Phys. Rev. Lett. 124, 211102 (2020); Q.Yan on behalf of AMS, Presented at this conference, PoS(ICHEP2020) 616.

[15] S. Thoudam and J. R. Hörandel, Mon. Not. R. Astron. Soc. 435, 2532 (2013). P. Blasi, E. Amato, P. D. Serpico, Phys. Rev. Lett. 109, 061101 (2012); A. E. Vladimirov, G. Jóhannesson, I. V. Moskalenko, T. A. Porter, Astrophys. J. 752, 68 (2012).

[16] F. Giovacchini on behalf of AMS, Presented at this conference, PoS(ICHEP2020) 594. J. Wei on behalf of AMS, Presented at this conference, PoS(ICHEP2020) 614. F. Dimiccoli on behalf of AMS, Presented at this conference, PoS(ICHEP2020) 591. 